\title{
MALDI-TOF MS for rapid identification of Mycobacterium species in liquid culture media
}

\begin{abstract}
The differentiation of Mycobacterium tuberculosis complex and NTM is important for the appropriate treatment and overall patient management. In this study, we have evaluated MALDI-TOF MS for species level identification of clinical mycobacterial isolates in comparison with gold standard hsp65 gene sequencing. Species level identification of mycobacteria by conventional methods is labor intensive and restricted to a few laboratory setups. A total of 60 mycobacteria isolates, comprising of 56 archived isolates and 4 CAP proficiency isolates were tested in this study. MALDI-TOF MS and hsp65 gene sequencing showed agreement in 52 of 60 isolates $(86.67 \%)$. All the 4 CAP proficiency panel isolates gave expected results by both the methods. Of the 08 unidentified isolates, 5 were not present in VITEK-MS Database v.3.0, but hsp65 gene sequencing could identify them as $M$. paraense $(\mathrm{N}=2)$, . parascrofulaceum $(\mathrm{N}=1)$ and $M$. $s p \mathrm{FI}-06083(\mathrm{~N}=2)$. The remaining 3 isolates that were unidentifiable on VITEK-MS turned out to be mixed isolates as revealed by hsp65 gene sequencing. This was possibly due to contamination of culture upon storage. Overall, MALDI-TOF MS was found to be rapid and cost-effective system for the identification of majority of mycobacterial species. DNA sequencing can be considered as a complementary tool for speciation of rare species and other discrepant isolates.
\end{abstract}

Volume 6 Issue 2 - 2018

\author{
Shamma Shetye,' Pratiksha Chheda, ${ }^{2}$ Dipti \\ Walke, ${ }^{3}$ Sunmeet Matkar, ${ }^{2}$ Usha Mukundan ${ }^{4}$ \\ 'Department of Microbiology, Metropolis Healthcare Ltd., India \\ ${ }^{2}$ Department of Molecular Pathology, Metropolis Healthcare \\ Ltd., India \\ ${ }^{3}$ Department of Microbiology, Metropolis Healthcare Ltd., India \\ ${ }^{4}$ HOD-Department of Biotechnology, R.J.College, India
}

Correspondence: Shamma Shetye, MD, HOD-Department of Microbiology, Metropolis Healthcare Ltd., India, Email: shamma.shetye@gmail.com

Received: February 26, 2018| Published: March 20, 2018

Keywords: MALTI-TOF MS, Mycobacterium, hsp gene sequencing, liquid culture

The genus Mycobacterium consists of at least more than 150 recognized species (LPSN database last accessed on 13th September 2017) [http://www.bacterio.net/index.html]. Pathogenicity ranges from environmental saprophytes to the pathogenic and health hazard M. tuberculosis. Tuberculosis is one of the leading causes of morbidity and mortality with 1.8 million deaths and 10.4 million new cases every year worldwide and continues to be a public health problem globally. ${ }^{1}$ A number of the environmental mycobacterium species, collectively known as non-tuberculosis mycobacteria (NTM), are responsible for many opportunistic infections that are increasingly common among immune-compromised individuals. Speciation is important to ascertain the clinical significance of the mycobacteria isolated. Also, differentiation of the Mycobacterium Tuberculosis Complex (MTC) and NTM has become important due to the rise of infections and antimicrobial resistance in this genus.

Conventional methods of identification include growth characteristics, temperature and rate of growth, colony pigmentations, biochemical reactivity etc. These are laborious, difficult to perform and require additional time after the culture flags positive hence most laboratories find these difficult to adopt in routine settings. Nowadays, positive cultures are identified as MTC based on smear morphology and methods like immunochromatography (MPT64 antigen) wherein NTM is a diagnosis of exclusion. Post-culturing, reliable molecular line probe assay like Geno Type CM/AS (Hain test) enables rapid (within 24hours) and reliable speciation of 32 clinically relevant mycobacteria and differentiates MTC from NTMs. Though reliable and highly sensitive, the test is laborious, requires skilled technologists, is expensive and therefore is mostly available in reference laboratories. Also, the assay is able to detect only a limited number of specified targets against which the probes are directed.

With the rise of opportunistic pathogens and with several new species being named annually, the need to have a rapid and reproducible identification method for a wider range of Mycobacterium species is evident. Recent developments in mass spectrometry (MS) have made it possible to use matrix-assisted laser desorption ionization-time-offlight MS (MALDI-TOF MS) or MALDI to aid in the identification of microorganisms. In recent years, several reports have shown the feasibility of matrix-assisted laser desorption ionization-time of flight mass spectrometry (MALDI-TOF MS) for the identification of microorganisms in routine clinical microbiology laboratories. ${ }^{2}$ Qualified spectra for the identification of various bacteria have been determined, but the methods for identifying mycobacteria species are new. ${ }^{3-5}$ Most studies utilizing MALDI-TOF MS for NTM identification have used culture collections rather than fresh positive cultures from clinical specimens. A PCR-sequencing method based on hsp65, the gene encoding the 65-kilodalton heat shock protein, has also been used extensively for differentiating $\mathrm{M}$. tuberculosis from other NTM members.

The current study was aimed to demonstrate the utility of MALDITOF MS for the identification of clinical mycobacterial isolates in a large microbiology laboratory. Further the results of MALDI-TOF MS were validated using gold standard hsp65 gene sequencing. To the best of our knowledge, this is the first comprehensive study from India characterizing various Mycobacterium species by MALDI-TOF MS and sequencing on isolates grown in liquid culture.

A total of 56 archived isolates and 4 CAP proficiency isolates were analyzed. For MALDI TOF MS analysis mycobacteria positive liquid culture $(3 \mathrm{ml})$ was collected in a $5-\mathrm{ml}$ vortex bottle. Vortex bottle was centrifuged at $3000 \mathrm{~g}$ for $10 \mathrm{~min}$. After discarding supernatant, pellet was re-suspended in $500 \mu \mathrm{L}$ of $70 \%$ ethanol. The suspension was transferred to a vial containing $0.5 \mathrm{~mm}$ glass beads. "Bead beat" treatment was given for $5 \mathrm{~min}$ and incubated at room temperature for $10 \mathrm{~min}$. After vortexing and transferring to fresh micro tube, it was centrifuged at $4000 \mathrm{~g}$ for $2 \mathrm{~min}$. The pellet was re-suspended in $10 \mu \mathrm{L}$ 
of $70 \%$ formic acid followed by addition of $10 \mu \mathrm{L}$ of $100 \%$ acetonitrile and spinning at $4000 \mathrm{~g}$ for $2 \mathrm{~min}$. One $\mu \mathrm{L}$ of the supernatant was spotted on a MS target slide and subjected to air-dry. The spot was overlaid with $1 \mu \mathrm{L}$ of CHCA ( $\alpha$-cyano-4-hydroxycinnamic acid) matrix solution followed by air-dry. The slide was processed using the VITEK-MS instrument. Mass spectra were analyzed using automatic database within MYLA software (Biomerieux, France). IVDv3.0 database of VITEK-MS was used in our study.

For Molecular identification, DNA was extracted from $200 \mu \mathrm{L}$ of liquid culture using QIAamp Blood Mini kit (Qiagen) and suspended in $200 \mu \mathrm{L}$ of elution buffer. An amplicon corresponding with $65-\mathrm{kDa}$ heat shock protein gene (hsp65) was amplified using primers TB11 [5'-ACCAACGATGGTGTGTCCAT] and TB12 [5'-CTTGTCGAACCGCATACCCT] described by Telenti PCR was performed in $20 \mu \mathrm{L}$ containing KAPA Ready Mix (Kapa Biosciences). $10 \mathrm{pmol}$ of each primer and $3 \mu \mathrm{L}$ of DNA prep. The PCR consisted of $95^{\circ} \mathrm{C}$ for $5 \mathrm{~min}$ followed by 35 cycles at $94^{\circ} \mathrm{C}$ for $15 \mathrm{~s}, 60^{\circ} \mathrm{C}$ for $30 \mathrm{~s}$, $72^{\circ} \mathrm{C}$ for $60 \mathrm{~s}$ and an extension at $72^{\circ} \mathrm{C}$ for $7 \mathrm{~min}$. PCR was followed by bidirectional sequencing on 3500Dx Genetic Analyzer (Applied Biosystems). The mycobacterial sequence was compared with other mycobacterial sequences and species showing $>99 \%$ homology through NCBI Gen Bank database.

A total of 60 isolates, representing 17 species belonging to 8 groups of Mycobacteria were used to assess the performance and clinical validity of the MALDI-TOF MS system. These isolates were grown in mycobacterium growth indicator tubes (MGIT), the media commonly used for mycobacteria isolation in corresponding laboratory. In order to validate our results, the same clinical isolates were subjected to hsp65 gene sequencing separately.

MALDI-TOF and hsp65 genes sequencing showed identical results in 52/60 (86.67\%) of cases (Table 1) and $8(13.3 \%)$ isolates remained unidentified. All the four CAP proficiency isolates i.e. each one of M. gordonae, M. xenopi, M. marinum and $M$. intracellulare gave expected results by both the methods. Of the eight unidentified isolates, five of the isolates were identified as $M$. paraense $(\mathrm{N}=2)$, M. parascrofulaceum $(\mathrm{N}=1)$ and $M . s p$ FI-06083 $(\mathrm{N}=2)$ by hsp65 gene sequencing. These isolates remained unidentified by mass spectrometry since they were not recognized by the IVDv3.0 database of VITEK-MS (Table 1). The remaining 3 unidentified isolates provided mixed sequence data indicating presence of mixed culture. Further analysis of sequence data identified presence of $M$. intracellulare + M. parascrofulaceum, M. abscessus + M. fortuitum and M. abscessus $+M$. intracellulare) respectively (Table 2). Culturing microorganisms as pure culture is prerequisite for MALDI-TOF MSbased identification since cross-contaminated or mixed cultures can lead to ambiguous and confusing results. Ferreira and coworkers have reported the inability of MALDI-TOF MS to accurately identify mixed bacteria. ${ }^{6}$

Another observation corresponds with group of $M$. fortuitum. While hsp65 gene sequencing accurately identified seven cases of M. fortuitum and each one of M. porcinum, M. peregrinum and M. conceptionense, MALDI-TOF MS collectively identified all of these 10 microorganisms as $M$. fortuitum group. The members of $M$. fortuitum clade include $M$. fortuitum, M. peregrinum, $M$. senegalese, M. mageritense, M. septicum, M. alvei, M. houstonense, M. boenickei, M. conceptionense, M. porcinum, M. neworleansense and $M$. brisbanense. These are rapidly-growing opportunistic human pathogens that cause a wide spectrum of clinically significant symptoms. M. porcinum, a rapidly-growing pathogenic species of human has been reported to cause post-traumatic or post-surgical wound infections, osteomyelitis, and catheter-related infections. ${ }^{7}$ $M$. conceptionense has been reported to cause infection after breast implant surgery as well as a subcutaneous abscess in an immunecompetent patient. ${ }^{8}$ Reports also reveal that they are resistant to most first-line anti-tuberculous agents. ${ }^{9}$ It is therefore important for practitioners to be aware of these rapidly-growing opportunistic and pathogenic microorganisms as possible etiological agents.

Table I Identification of Mycobacterial species through MALDI-TOF MS system and hsp65 gene sequencing

\begin{tabular}{llll}
\hline Organism & Total & $\begin{array}{l}\text { Results of MALDI- } \\
\text { TOF MS system }\end{array}$ & $\begin{array}{l}\text { Results of hsp65 } \\
\text { gene sequencing }\end{array}$ \\
\hline M. abscessus & 18 & 18 & 18 \\
M. avium & $\mathrm{I}$ & $\mathrm{I}$ & $\mathrm{I}$ \\
M. chelonae & $\mathrm{I}$ & $\mathrm{I}$ & $\mathrm{I}$ \\
M. fortuitum & $\mathrm{I} 0$ & $\mathrm{1} 0$ & $10^{*}$ \\
M. gordonae & 2 & 2 & 2 \\
M. intracellulare & 7 & 7 & 7 \\
M. kansasii & 2 & 2 & 2 \\
M. lentiflavum & $\mathrm{I}$ & $\mathrm{I}$ & $\mathrm{I}$ \\
M. marinum & $\mathrm{I}$ & $\mathrm{I}$ & $\mathrm{I}$ \\
M. scrofulaceum & $\mathrm{I}$ & $\mathrm{I}$ & $\mathrm{I}$ \\
M. simiae & 5 & 5 & 5 \\
M. smegmatis & $\mathrm{I}$ & $\mathrm{I}$ & $\mathrm{I}$ \\
M. tuberculosis & $\mathrm{I}$ & $\mathrm{I}$ & $\mathrm{I}$ \\
M. xenopi & $\mathrm{I}$ & $\mathrm{I}$ & $\mathrm{I}$ \\
M. paraense & 2 & 0 & 2 \\
M. & $\mathrm{I}$ & 0 & $\mathrm{I}$ \\
parascrofulaceum & & 0 & 2 \\
M. sp Fl-06083 & 2 & 0 &
\end{tabular}

*hsp65 gene sequencing further identified $M$. porcinum, $M$. peregrinum and $M$. conceptionense from $M$. fortuitum group of Mycobacteria.

Table 2 Mycobacterial species identified in the study

\begin{tabular}{llll}
\hline Sr. no & Group & Species & Count \\
\hline I & Mycobacterium. Chelonae & M. abscessus & 18 \\
& & M. chelonae & 1 \\
2 & $\begin{array}{l}\text { Mycobacterium avium } \\
\text { complex (MAC) }\end{array}$ & M. avium & I \\
& M.intracellulare & 7 \\
3 & Mycobacterium fortuitum & M. fortuitum & 10 \\
4 & Mycobacterium gordonae & M. gordonae & 2 \\
5 & Mycobacterium kansasii & M. kansasii & 2 \\
6 & Mycobacterium simiae & M. simiae & 5 \\
& & M. lentiflavum & 1 \\
7 & $\begin{array}{l}\text { Mycobacterium tuberculosis } \\
\text { complex (MTC) }\end{array}$ & M. tuberculosis & 1 \\
& & &
\end{tabular}


Table Continued

\begin{tabular}{llll}
\hline Sr. no & Group & Species & Count \\
\hline 8 & Other Mycobacteria group & M. marinum RI & I \\
& & M. scrofulaceum RII & I \\
& & M. smegmatis & I \\
& & M. xenopi & I \\
\hline
\end{tabular}

Although the number of isolates in our study was not large enough $(\mathrm{N}=60)$ and the number of species was lower $(\mathrm{N}=17)$, our results show that identification using MALDI-TOF is reliable in majority of clinically-relevant species of NTM $(M$. avium, $M$. intracellulare, $M$. abscessus, M. chelonae, M. fortuitum, M. kansasii, M. scrofulaceum). Several studies have documented the effect of culture time on the accuracy of identifying mycobacterial species. ${ }^{10,11}$ Few authors have found dissimilarities in identification depending upon the culture media used, the culture growth time, the extraction protocol applied to the mycobacteria, or the library (i.e. Bruker Biotyper and Vitek MS databases) used. ${ }^{10-12}$ Our results were obtained from clinical isolates, where most of them were assayed within 2-5 days of growth in MGIT liquid medium. ${ }^{13}$

Our study demonstrated that besides low cost and accuracy, MALDI-TOF MS procedure involves little handling and few working hours, which reduces the potential risk of infections caused by mycobacteria in the laboratory. MALDI-TOF MS could be regarded as an alternate diagnostic tool for identification and differentiation of clinical isolates of Mycobacterium. Sequence-based identification remains a complimentary approach for evaluation of unidentified isolates. In order to have comprehensive clinical diagnosis, Sanger sequencing and /or Next Generation Sequencing technology can be utilized by clinical laboratories.

\section{Acknowledgements}

None.

\section{Disclosure Statement}

The authors declare that they have no conflicts of interest.

\section{References}

1. Raviglione M, Sulis G. Tuberculosis 2015: burden, challenges and strategy for control and elimination. Infect Dis Rep. 2016;8(2):6570.

2. Clark AE, Kaleta EJ, Arora A, et al. Matrix-assisted laser desorption ionization-time of flight mass spectrometry: a fundamental shift in the routine practice of clinical microbiology. Clin Microbiol Rev 2013;26(3):547-603.
3. Neuschlova M, Vladarova M, Kompanikova J, et al. Identification of mycobacterium species by MALDI-TOF mass spectrometry. Adv Exp Med Biol. 2017;1021:37-42.

4. Saleeb PG, Drake SK, Murray PR, et al. Identification of mycobacteria in solid-culture media by matrix-assisted laser desorption ionization-time of flight mass spectrometry. J Clin Microbiol. 2011;49(5):1790-1794.

5. Bensi EPA, Panunto PC, Ramos M. Incidence of tuberculous and nontuberculous mycobacteria, differentiated by multiplex PCR, in clinical specimens of a large general hospital. Clinics (Sao Paulo). 2013;68(2):179_ 183.

6. Ferreira L, Sanchez-Juanes F, Munoz-Bellido JL, et al. Rapid method for direct identification of bacteria in urine and blood culture samples by matrix-assisted laser desorption ionization time-of-flight mass spectrometry: intact cell vs. extraction method. Clin Microbiol Infect. 2011;17(7):1007-1012.

7. Wallace RJ, Brown-Elliott BA, Wilson RW, et al. Clinical and laboratory features of mycobacterium porcinum. J Clin Microbiol. 2004;42(12):56895697.

8. Thibeaut S, Levy P, Pelletier M, et al. Mycobacterium conceptionense infection after breast implant surgery, France. Emerg Infect Dis. 2010;16(7):1180-1181

9. Pang H, Li G, Wan L, et al. In vitro drug susceptibility of 40 international reference rapidly growing mycobacteria to 20 antimicrobial agents. Int $J$ Clin Exp Med. 2015;8(9):15423-15431.

10. Lotz A, Ferroni A, Beretti J, et al. Rapid dentification of mycobacterial whole cells in solid and liquid culture media by matrix-assisted laser desorption ionization-time of flight mass spectrometry. J Clin Microbiol. 2010;48(12):4481-4486.

11. Mather CA, Rivera SF, Butler-Wu SM. Comparison of the bruker biotyper and vitek MS matrix-assisted laser desorption ionization-time of flight mass spectrometry systems for identification of mycobacteria using simplified protein extraction protocols. J Clin Microbiol. 2014;52(1):130 138 .

12. Buchan BW, Riebe KM, Timke M, et al. Comparison of MALDI-TOF MS with HPLC and nucleic acid sequencing for the identification of mycobacterium species in cultures using solid medium and broth. Am J Clin Pathol. 2014;141(1):25-34.

13. Parte AC. LPSN-- list of prokaryotic names with standing in nomenclature. Nucleic Acids Res. 1997;42:D613-D616. 